\title{
Fuentes de conocimiento e imágenes de la innovación en micro y pequeñas empresas de turismo: agencias de viajes y hoteles en Bogotá y Pereira
}

\section{Knowledge sources and images of innovation in micro and small-sized tourism enterprises: travel agencies and hotels in Bogotá and Pereira}

\author{
María Eugenia Morales-Rubiano' \\ Carolina Ortiz-Riaga ${ }^{2}$ \\ Yenni Viviana Duque-Orozco ${ }^{3}$ \\ Paola Andrea Plata-Pacheco ${ }^{4}$ \\ Recibido: agosto 29 de 2016 \\ Aceptado: noviembre 24 de 2016
}

\section{Resumen}

El objetivo del documento es identificar las fuentes de conocimiento a las que acuden micro y pequeños empresarios del sector turismo y si estas son utilizadas como base para generar innovación. Se realizaron entrevistas semi estructuradas a los gerentes de seis hoteles y nueve agencias de viajes en las ciudades de Bogotá y Pereira. Los resultados evidencian que los empresarios acuden a fuentes de conocimiento propio, familiar y del mercado, dejando de lado el conocimiento especializado proveniente de procesos de investigación y/o de entidades de orden regional y nacional. También se aprecia que las imágenes que se han construido sobre la innovación están relacionadas con dimensiones como: prácticas diferenciadoras, novedad o mejora, implementación de herramientas tecnológicas y validación de la innovación por el mercado. Se concluye que el uso limitado de las fuentes no logra modificar la base de recursos de manera sustancial, por lo tanto, dificulta la posibilidad de generar innovación.

Palabras clave: fuentes de conocimiento, imágenes de la innovación, sector turismo.

\begin{abstract}
The aim of this paper is to identify the sources of knowledge to entrepreneurs attending the tourism sector and establish whether the sources are used as a basis to generate innovation. Semi-structured interviews to six hotel managers and nine travel agencies from the tourism sector in the cities of Bogota and Pereira were made. The results show that employers turn to their own sources of knowledge, their family and the market, leaving aside the specialized knowledge from research processes and / or from regional or national institutions order. It is also appreciated that the images that they have been built about innovation are related to dimensions such as differentiating practices, novelty or improvement, implementation of technological tools and validation of innovation by the market. It is concluded that the limited use of sources fails to modify the resource base substantially, therefore, this makes it difficult to generate innovation.
\end{abstract}

Keywords: sources of knowledge, images of innovation, tourism sector.

\footnotetext{
1 Administradora de Empresas, Magíster en Administración, Universidad Militar Nueva Granada, Colombia. E-mail: maria.morales@ unimilitar.edu.co

2 Psicóloga, Magíster en Educación, Universidad Militar Nueva Granada, Colombia. E-mail: maria.ortiz@unimilitar.edu.co

3 Administradora de Empresas, Magíster en Gestión de Organizaciones, Universidad Militar Nueva Granada, Colombia. E-mail: yenni. duque@unimilitar.edu.co

4 Administradora de Empresas, Universidad Militar Nueva Granada, Colombia. E-mail: andreaplatap@gmail.com
} 


\section{Introducción}

Con frecuencia los estudios sobre innovación se realizan teniendo como referente las grandes empresas, pero es claro que la mayoría de las empresas en el ámbito regional son Micro, pequeñas y medianas empresas, Mipyme, y que estas son fundamentales para el desarrollo económico de los países, por su contribución al empleo y su aporte al Producto Interno Bruto-PIB. En Colombia, este tipo de empresas representan el $96 \%$ de las unidades económicas y el $76 \%$ del empleo (Cantillo, 2011).

De acuerdo con Velasco, Zamanillo y Intxaurburu (2007) a pesar de que hace más de un siglo se reconoce que la innovación es un factor de competitividad en las organizaciones, aún no se ha logrado establecer un proceso de implementación generalizada y práctica, que además reúna la complejidad que implica llevar a cabo innovación en una organización.

Este trabajo tiene como propósito establecer las fuentes de conocimiento a las que acuden los empresarios de micro y pequeñas empresas del sector turismo: agencias de viaje y hoteles, y si estas son utilizadas como base para generar innovación. También se busca identificar las imágenes de la innovación que estos asumen como válidas en su contexto particular. Es así, que el documento contribuye al reconocimiento de las dificultades que presentan las micro y pequeñas empresas del sector turismo para insertarse en un mercado altamente competitivo y liderado por grandes competidores que han desarrollado capacidades para generar valor agregado y adaptabilidad a un contexto dinámico.

A continuación se presenta una revisión de literatura sobre algunas concepciones de la innovación, el conocimiento como ventaja competitiva, sus fuentes y cómo las micro y pequeñas empresas acuden a estas. Posteriormente, se presentan la metodología del estudio y los resultados obtenidos. Por último, se exponen las reflexiones finales.

\section{Marco teórico y metodología}

\subsection{Concepciones sobre innovación}

Gros y Lara (2009) sostienen que la palabra innovación está de "moda" y por ello puede utilizarse en diferente forma y con múltiples significados según el contexto. Kohler y González (2014), sostienen que son sonidos que se repiten en cualquier espacio en donde se haga referencia a empresas, a competitividad o a competencia (Rodríguez, 2010), muchas veces careciendo de significado. Lo que es compartido, para las organizaciones en general es su utilización como un elemento de valor y de diferenciación (Gros., \& Lara, 2009).

A pesar de sus diversos significados, la innovación en la cotidianidad se asocia a la necesidad de mejorar las condiciones materiales o simbólicas de las personas. En este sentido se asocia con la necesidad de las personas de vivir mejor. "Podemos entender la innovación como aquella idea, objeto o práctica percibida como nueva por un individuo o individuos, que intenta introducir mejoras en relación a los objetivos deseados, que por naturaleza tiene una fundamentación, y que se planifica y delibera" (Rodríguez., \& Jiménez, 2010). Esta, junto con otras definiciones consultadas, tiene un denominador común: realizar cambios con la finalidad de mejorar.

Al respecto se identifican tres dimensiones que han imperado y marcado los usos de la innovación: una económica, una social y una educativa. Sin embargo, es tan fuerte y generalizado el uso de la dimensión económica de la innovación, que la Real Academia Española de la Lengua (2015, párr. 1) sostiene que la innovación es la "Creación o modificación de un producto, y su introducción en un mercado". Esto es así desde inicios del siglo XX y desde entonces la dimensión económica de la innovación se instauró como la principal.

Teniendo en cuenta el propósito de este artículo, se tomará como eje central las innovaciones económicas, las cuales desde Schumpeter en 1911, 
se vincularon inicialmente al espíritu emprendedor, como factor que potencia el crecimiento de las empresas, y por tanto el desarrollo económico; si bien, existen factores externos que afectan a la organización, se considera al empresario como el motor fundamental (Schumpeter, 1911, citado en Viega, 2011). En este sentido, la definición de mayor aceptación y funcionalidad en el campo empresarial es la promulgada por la Organización para la Cooperación y el Desarrollo Económico OCDE en el Manual de Oslo, en donde afirma que la innovación es "la introducción de un nuevo, o significativamente mejorado, producto (bien o servicio), de un proceso, de un nuevo método de comercialización o de un nuevo método organizativo, en las prácticas internas de la empresa, la organización del lugar de trabajo o las relaciones exteriores" (OCDE., \& EUROSTAT, 2006).

De esta definición se pueden extraer conceptos clave como novedad, mejoramiento significativo y la aplicabilidad de la innovación en diferentes aspectos de la empresa. Así mismo, diversos autores han identificado las tipologías de innovación empresarial en términos de producto, procesos, mercadeo y organizacional (Baba, 2012; Baregheh., Rowley., Sambrook., \& Davies, 2012; OCDE., \& EUROSTAT, 2006). La innovación de producto (bien o un servicio), implica que se genera o se mejora una característica o uso, cambio en componentes, materiales, tecnologías u otras funcionalidades agregadas; la innovación en proceso, hace referencia a la producción o distribución, reflejado en cambios significativos en técnicas, materiales o tecnologías utilizadas; la innovación en mercadotecnia, se relaciona con los métodos de comercialización, contemplando desde el cambio en el envasado, hasta su promoción o "tarificación"; finalmente, la innovación de organización, es el cambio o introducción de nuevas prácticas en el lugar de trabajo o las relaciones de la empresa con externos (OCDE., \& EUROSTAT, 2006).

Toda innovación debe incluir un elemento de novedad que puede ser: nuevo para la empresa, nue- vo para el mercado o nuevo para el mundo entero. En este sentido:

"La exigencia mínima para reconocer una innovación es el hecho de ser nueva para la empresa. Un producto, un proceso, un método de comercialización o un método de organización pueden haber sido aplicados ya por otras empresas pero si son nuevos para la empresa en cuestión (o, en el caso de los productos y procesos: significativamente mejorado), se trata de una innovación para esta empresa. (OECD., \& EUROSTAT, 2006, 69-70)"

En razón de lo anterior, las innovaciones pueden ser de alcance local, nacional o internacional, lo cual muestra que se puede empezar por hacer las cosas de manera diferente, generando cambios en los productos, servicios o procesos internos de la empresa. Así mismo, la innovación según el grado puede ser radical con o sin patente, si es algo totalmente nuevo; incremental, si se hacen pequeños cambios dirigidos a mejorar la funcionalidad, eficiencia y/o satisfacción del usuario o cliente; y decremental, si se le quitan atributos o cualidades al producto para poderlo llevar a un mercado de menores ingresos (OECD., \& EUROSTAT, 2006; Ruelas, 2004).

Partiendo entonces de los procesos para generar innovación empresarial, Martínez (2006) indica que la innovación busca el uso y la capitalización del conocimiento. Por tanto la capacidad para acceder a nuevas fuentes de conocimiento y los procesos de aprendizaje que se desprenden seguidamente, conllevan a que los empresarios perciban la importancia de acceder a nueva información de diversas fuentes y procesarla con el propósito de generar cambios o mejoras significativas en sus productos, procesos, métodos de mercadeo u de organización, como fuente de ventajas competitivas en el mercado.

2.2 Conocimiento e innovación para la creación de ventajas competitivas 
La generación de ventajas competitivas puede ser vista desde diversos enfoques. Desde la perspectiva basada en la posición competitiva en el sector (Porter, 1988), hasta una perspectiva de gestión intra organizacional a partir del enfoque basado en recursos (Čiutienè., \& Thattakath, 2014; Vivas, 2013). Pese a que son enfoques diferentes, contemplan paralelamente el conocimiento que provee el entorno y el que puede generar la organización internamente.

A partir de estos enfoques se evidencia que un elemento en común que ha determinado la posición competitiva, es el conocimiento como recurso clave y estratégico de una empresa (Abeson., \& Taku, 2009; Takeuchi, 2013). Este provee de una diferenciación a la empresa, que sobrepasa las que se pueden generar a partir de "cadenas de valor, actividades, recursos y competencias" (Takeuchi, 2013).

Consecuentemente, al adquirir conocimiento, se conciben procesos de aprendizaje al interior de la empresa, que permiten incrementar el desempeño en los procesos organizacionales. Zollo $\&$ Winter (2002), identifican tres mecanismos de aprendizaje para la generación de capacidades de innovación: "1) acumulación de experiencias, 2) articulación del conocimiento y 3) codificación de conocimiento". De manera que los autores, plantean el aprendizaje organizacional como base para las capacidades para la innovación, necesarias en el sentido que provee a la empresa la habilidad para responder a los cambios del entorno competitivo (Zollo., \& Winter, 2002; Vivas, 2013).

La capacidad para la innovación es definida como: "la capacidad de una organización para crear, extender y modificar su base de recursos a propósito" (Helfat, C., Finkelstein, S., Mitchell, W., Peteraf, M., Singh, H., Teece, D., \& Winter, S, 2007), es decir que al reformar la base de recursos y capacidades, la organización podrá proponer nuevas estrategias para responder al cambio o inclusive generarlo.
Si se entiende el conocimiento como un factor estratégico, y a los procesos de aprendizaje como generadores de capacidades para la innovación, en este contexto será conveniente estudiar el primer aspecto, el de adquirir conocimiento desde diversas fuentes, como un acercamiento inicial a la generación de competitividad.

2.3 Fuentes de conocimiento para la creación de capacidades para la innovación

Adquirir conocimiento como primera etapa en la generación de procesos de aprendizaje organizacional, parte del supuesto de que la empresa está en constante interacción con diversas fuentes de información, que permiten identificar con mayor precisión los cambios en el entorno y dar respuesta a estos (Abeson., \& Taku, 2009; Robinson., \& Anton, 2011). Existen fuentes que se derivan de la generación de rutinas y la acumulación de experiencias dentro de la organización (Zollo., \&Winter, 2002), mientras que otras, aprovechan el mercado de conocimiento, algunas veces a través de la intervención de agentes externos a la organización (García., Pelechano., \& Navas, 2007).

En este punto es importante retomar el planteamiento que realizan Zollo \& Winter (2002), frente a la adquisición de conocimiento por medio de diversas fuentes, ya que muestran que son esencialmente un "estímulo que permite iniciar propuestas para la modificación de rutinas" (p. 350). Plantean como ejemplo, el conocimiento de las necesidades del cliente, que siendo un factor clave de toda estrategia empresarial, por sí solo no crea o modifica rutinas. En este sentido, se requiere que la organización genere procesos internos para asimilar dicho conocimiento: apropiarlo, transformarlo y explotarlo. En este sentido, la literatura muestra que uno de los primeros pasos consiste en un proceso de exploración del conocimiento (Acosta., \& Fischer, 2013; Vivas, 2013).

La exploración se entiende entonces como"la búsqueda del conocimiento que puede llegar a ser conocido" (Levinthal., \& March, 1993; en Acosta., 
\& Fischer, 2013); dicha búsqueda implica procesos internos de creatividad, imaginación, descubrimiento y de toma intencional de riesgos (Abeson, \&Taku, 2009), al igual que procesos de adquisición de información de terceros (García, Pelechano, \& Navas, 2007). La necesidad de toda empresa, en contar con diversas fuentes de conocimiento, radica en las respuestas que puedan dar a los retos del entorno cambiante. Si por un lado el reto se puede solucionar con capacidades ya adquiridas, habrá que utilizar conocimiento interno; sin embargo, cuando no se logra una solución efectiva, se debe acudir al exterior para obtener un mejor entendimiento del entorno (García, Pelechano, \& Navas, 2007). Por tanto la literatura muestra diversas fuentes de conocimiento, útiles para las empresas desde diferentes perspectivas y que se pueden convertir en uno de sus factores de competitividad (Abeson, \& Taku, 2009; García, Pelechano, \&
Navas, 2007; González, 2011; Othman, Amaraa, \& Landrya, 2012; Robinson, \& Anton, 2011).

De acuerdo con los planteamientos de Othman, Amaraa y Landrya (2012), se presenta una clasificación de las fuentes según el origen del conocimiento, de manera que se tienen: fuentes del mercado, fuentes de investigación, fuentes de información general disponible y fuentes regionales y nacionales; adicionalmente a este planteamiento, se pueden considerar dos nuevas categorías, entendidas como fuentes familiares y relacionales a partir de lo descrito por Abeson y Taku (2009) y fuentes propias, entendiéndose como el conocimiento que se obtiene a partir de las trayectorias de los empleados y del gerente o dueño (González, 2011). La tabla 1 muestra las fuentes de conocimiento que se consideran para este estudio.

\begin{tabular}{|c|c|c|c|}
\hline \multirow{4}{*}{ Fuentes del mercado } & \multicolumn{2}{|l|}{ Proveedores } & \multirow{2}{*}{ Universidades } \\
\hline & Clientes & Fuentes de & \\
\hline & Competidores & investiga- & Centros de transferencia tecnológica \\
\hline & Firmas relacionadas & ción & Bases de datos \\
\hline \multirow{7}{*}{$\begin{array}{l}\text { Fuentes de información } \\
\text { general y especializada }\end{array}$} & Firmas de consultoría & & Documentos propiedad intelectual \\
\hline & $\begin{array}{l}\text { Ferias y exhibiciones } \\
\text { Internet }\end{array}$ & Fuentes & $\begin{array}{l}\text { Agencias y laboratorios de investiga- } \\
\text { ción }\end{array}$ \\
\hline & Capacitaciones especializadas & regionales y & Agremiaciones \\
\hline & Redes profesionales & & Cámaras de comercio \\
\hline & Revistas especializadas & Fuentes & Familia \\
\hline & Blogs & familiares y & Colegas \\
\hline & Entidades gubernamentales & relacionados & Amigos \\
\hline \multirow[t]{2}{*}{ Fuentes propias } & & Empleados & \\
\hline & Empresario, gerente & & \\
\hline
\end{tabular}

Tabla 1. Fuentes de conocimiento según su origen (basada en: Abeson \& Taku, 2009; González, 2011; Othman, Amaraa \& Landrya, 2012).

Como se aprecia en la tabla 1, son diversas las fuentes y tipo de conocimiento que tienen a disposición las empresas, y reconociendo que no todas cuentan con los mismos recursos para aprovechar el conocimiento del entorno (Robinson, \& Anton, 2011), surge un especial interés en identifi- car cómo las Mipyme adquieren conocimiento del entorno dadas sus características particulares.

2.4 ¿Cómo adquiere el conocimiento una Mipyme?

Una vez descrita la importancia del conocimiento en una organización como fuente de ventaja com- 
petitiva y de las fuentes de conocimiento disponibles para las empresas, es necesario indicar las características particulares de dicha adquisición por parte de las empresas a estudiar.

Las Mipyme, de acuerdo con Yew y Aspinwall (2004), poseen características distintivas para la adquisición de conocimiento. Iniciando por su estructura, la cual al ser reducida, facilita los procesos de comunicación interna y por tanto el flujo de conocimiento entre empleados; así mismo, al estar centralizadas las decisiones en el gerente, que en ocasiones es el dueño, origina que el conocimiento que este posee, sea por su trayectoria personal, educativa o laboral o por la cercanía al conocimiento de familiares, amigos o colegas, influya directamente en la organización. Por otro lado, se percibe que la cercanía con el cliente, permite igualmente generar mejores relaciones, logrando un mejor entendimiento del mismo.

Según lo anterior, las Mipyme presentan una ventaja frente a la adquisición del conocimiento que generalmente proviene de fuentes propias y fuentes familiares y de relacionados. Sin embargo, el reto radica en balancear a cuántas fuentes acude y cómo las aprovecha (Othman, Amaraa., \& Landrya, 2012; Yew, \& Aspinwall, 2004).

2.5 Fuentes de conocimiento para las Mipyme en el sector turismo

El turismo, de acuerdo con la Organización Mundial del Turismo, OMT, (1991), es el conjunto de actividades realizadas por personas durante los viajes, en espacios situados fuera de su residencia habitual, por un período consecutivo que no excediese un año por motivos de ocio, negocios y otros. En el escenario del mercado turístico confluyen los operadores turísticos: p.ej. agencias de viajes, y la oferta turística dentro de la cual se encuentra el recurso de planta como lo es el alojamiento.

A nivel global, El sector turismo se visualiza como un sector de alto y rápido crecimiento. En especial el Reporte de Competitividad de Viajes y Turismo,
RCVT, (Foro Económico Mundial, 2015) indica que a pesar de que los países desarrollados tienen mejores condiciones para capturar la demanda de turismo global, los países emergentes y en desarrollo están creando una dinámica particular que se da por el incremento de turistas jóvenes, que a su vez impulsan el crecimiento del servicio en línea y a través de teléfonos celulares.

Considerando entonces la dinámica de crecimiento del sector, su estructura compleja e interdependiente entre diversas organizaciones, la perspectiva competitiva implica que las empresas, en especial las Mipyme, utilicen el conocimiento a favor de sus estrategias de posicionamiento y crecimiento (González, 2011). Así lo demuestran los retos que enfrentan para su permanencia en el mercado, entre los que se incluyen la popularización del internet, las nuevas tecnologías de auto reserva, la disminución de la lealtad de los clientes y la sobreoferta de servicios.

Entre las fuentes de conocimiento que se destacan para el sector turismo, resalta el papel de los empleados, que constituyen el medio para prestar el servicio en el sector, dado que están directamente relacionados con los clientes, de manera que en el empleado se logra condensar conocimiento relacionado con el cliente y el entorno, Es por esto que Ruizalba, Vallespín y Pérez, (2015) sugieren a las empresas del sector, generar una adecuada gestión del conocimiento de los empleados a partir de su retención y formación.

García (2009), identifica particularmente para el sector turismo, la necesidad de explorar diversas fuentes de conocimiento en el entorno. En primer lugar se menciona la necesidad de obtener información del mercado en tiempo real, ya que al ser el turismo un sector cambiante y dependiente de múltiples variables, la información oportuna incide en la toma de decisiones estratégicas; seguidamente la formación profesional, como medio para aprovechar la información disponible en la empresa; no basta con tener información, es necesario procesarla internamente y decidir a partir de ella; 
y en tercer lugar, la creación de redes de contacto, sea con proveedores, distribuidores o clientes, puesto que para enfrentar el reto de calidad con un equilibrio en el costo, se requiere de alianzas con las diversas partes interesadas. Es por esto que el sector turismo debe tratar de contar con fuentes fiables, accesibles y económicas.

En Colombia, el sector turismo es considerado como estratégico para impulsar el desarrollo económico y empresarial. Este sector hace parte del Programa de Transformación Productiva del Gobierno Nacional, que busca la generación de entornos más competitivos y empresas más fuertes y productivas. Se compone en su cadena de valor por más de trece mil empresas, representadas en un $49.79 \%$ por servicios de alojamiento y hospedaje, y en un $26.58 \%$ por agencias de viaje; con un menor porcentaje de participación se incluyen: los guías de turismo, $11.11 \%$, los establecimientos de gastronomía y similares, $5.18 \%$, las oficinas de representación turística, $2.43 \%$, y los operadores profesionales de congresos, ferias y convenciones, 1.91\% (Confecámaras, 2013).

Lo anterior dimensiona la importancia que los hoteles y las agencias de viaje representan para el sector, razón por la que esta investigación tomó como referencia estos dos eslabones del sector turismo. Las Mipyme del sector turismo en Colombia, tienen también la necesidad de obtener información del mercado en tiempo real, fortalecer la formación profesional de su personal y generar de redes de contacto para hacer frente a los retos de la evolución del sector, y adicionalmente mejorar los niveles de percepción de calidad del turista sobre los servicios ofrecidos.

En concordancia con lo anterior, un reto para las Mipyme del sector turístico en Colombia es definir fuentes de información que cumplan criterios de accesibilidad, fiabilidad y economía, que les permitan identificar en tiempo real información del entorno para generar procesos de aprendizaje y cambio organizacional que garanticen su perma- nencia en el mercado y posicionamiento competitivo frente a la oferta mundial.

\subsection{Metodología}

Esta investigación tiene un enfoque cualitativo y toma como método el estudio de caso a partir del estudio de quince empresas pertenecientes al sector turismo en los eslabones de alojamiento y agencias de turismo, en dos ciudades de Colombia priorizadas por pertenecer a dos de las regiones con mayor concentración de empresas del sector: centro con $28 \%$, y Antioquia y Eje cafetero con 21\% (Confecámaras, 2013).

En este contexto, "al ser el turismo un fenómeno social, es pertinente y científicamente válido estudiarlo aplicando el método de estudio de casos" (Peña, \& Serra, 2013,). Stake (2007) identifica tres tipos de estudio de caso de acuerdo con el número de unidades o entidades a considerar: i) Un solo caso o unidad de análisis; ii) Múltiples unidades de análisis o casos, que en primera instancia busca evaluar integralmente a cada uno por sí mismo, para después establecer tendencias y elementos comunes; iii) Múltiples casos cruzados o entrelazados, cuya diferencia respecto al tipo anterior es que desde el inicio se pretende revisar comparativamente los casos entre sí, buscando similitudes y diferencias.

La investigación realizada corresponde con el tercer literal, pues se trata de quince microempresas del sector turismo, que son objeto de estudio por eslabón y localización; con la posibilidad de establecer comparativamente los resultados obtenidos respecto a las fuentes de conocimiento e imágenes de la innovación.

Para identificar las organizaciones a estudiar se acudió a las fuentes de información del sector, como: Vitrina Turística ANATO 2015, Feria Internacional de Turismo FITUR 2015, Cámara de Comercio de Bogotá e Instituto Distrital de Turismo. Como resultado de la búsqueda en las anteriores fuentes; se identificaron micro y pequeñas empre- 
sas del sector turismo (agencias y alojamiento) en Bogotá y Pereira; luego se procedió a realizar un acercamiento para verificar el cumplimiento de las condiciones de operación y tamaño; y posterior- mente se invitaron a las que cumplían con los dos criterios a participar del proyecto, quedando 15 empresas objeto de estudio como se aprecia en la tabla 2.

\begin{tabular}{|c|c|c|c|c|c|c|}
\hline Ciudad & Empresa & $\begin{array}{c}\text { Persona } \\
\text { entrevistada }\end{array}$ & $\begin{array}{c}\text { Tipo } \\
\text { Empresa }\end{array}$ & $\begin{array}{c}\text { Tiempo } \\
\text { en el } \\
\text { mercado } \\
\end{array}$ & $\begin{array}{c}\text { Tamaño de la } \\
\text { empresa } \\
\text { ( } n^{\circ} \text { empleados) }\end{array}$ & $\begin{array}{l}\text { Alcance del } \\
\text { mercado }\end{array}$ \\
\hline \multirow{8}{*}{ Bogotá } & \multicolumn{6}{|c|}{ Agencia de viajes } \\
\hline & Over Visions Tours & Gerente general & Familiar & 34 años & Pequeña (20) & Nacional \\
\hline & Monarca & Gerente & Familiar & 11 años & Micro (4) & Local \\
\hline & Aramacao & Propietario & Familiar & 10 años & Micro (1) & Local \\
\hline & $\begin{array}{l}\text { Destinations by } \\
\text { Ana Gulden }\end{array}$ & Propietaria - Gerente & Familiar & $\begin{array}{l}2 \text { años y } \\
\text { medio }\end{array}$ & Micro (2) & Internacional \\
\hline & Hotel & & & & & \\
\hline & Hotel Fontanar & Propietarios & Familiar & 14 años & Micro (3) & Nacional \\
\hline & Hotel Casa Regis & Propietario- Gerente & Familiar & 12 años & Micro (7) & Internacional \\
\hline \multirow{11}{*}{ Pereira } & Agencia de viajes & & & & & \\
\hline & Destinos & $\begin{array}{l}\text { Gerente - Socia } \\
\text { minoritaria }\end{array}$ & Sociedad & 15 años & Micro (5) & Local \\
\hline & Disneys Tours & Gerente & Familiar & 16 años & Pequeña (14) & Nacional \\
\hline & Aerosueños & Gerente - Socia & Sociedad & 15 años & Micro (8) & Local \\
\hline & Travel Waze & Socias fundadoras & Sociedad & 1 año & Micro (3) & Local \\
\hline & $\begin{array}{l}\text { Colombiana de } \\
\text { Turismo }\end{array}$ & Propietario- Gerente & Familiar & 18 años & Micro (5) & Local \\
\hline & \multicolumn{6}{|l|}{ Hotel } \\
\hline & Hotel Los Cristales & Propietario & Familiar & 15 años & Micro (2) & Local \\
\hline & Hotel La Catalina & Propietarios & Familiar & 2 años & Pequeño (14) & Local \\
\hline & Hotel Cafeira & Gerente & Sociedad & $\begin{array}{l}2 \text { años y } \\
\text { medio }\end{array}$ & Pequeño (14) & Nacional \\
\hline & $\begin{array}{l}\text { Hotel Santo } \\
\text { Bambú }\end{array}$ & Propietarios & Familiar & 6 meses & Micro (5) & Nacional \\
\hline
\end{tabular}

Tabla 2. Características generales de las empresas estudiadas

El estudio se centra en el análisis de discurso, tanto de las trayectorias de la empresa como del empresario, entendiendo que los resultados empresariales no son independientes de los contextos de los sujetos que la integran. De manera tal que se realizaron quince entrevistas semiestructuradas a los gerentes o dueños de empresas del sector turismo: agencias de viaje y hoteles, en Bogotá y Pereira, como se muestra en la tabla 2.
Para el análisis de la información, en lo que se refiere a las fuentes de conocimiento, se elaboró una matriz en la que se sistematizaron las respuestas a partir de las categorías deductivas presentadas en la tabla 1; de otra parte, se tuvieron en cuenta categorías inductivas o emergentes para identificar las concepciones e imágenes sobre innovación, a partir de la frecuencia de aparición dentro del discurso de los entrevistados. 


\section{Resultados y discusión}

\subsection{Fuentes de conocimiento}

Las características generales de las empresas estudiadas muestran que la mayoría son microempresas, de índole familiar, con un promedio de 11 años en operación y con diferente alcance en el mercado. Entre la muestra, las empresas de Pereira son de propiedad familiar o de una sociedad, mientras que en Bogotá, en su totalidad, son empresas familiares. Igualmente en lo referente al tamaño Pereira tiene mayor representatividad en micro y pequeñas empresas, diferente a la muestra en Bogotá en donde se contempló una pequeña empresa y el resto micro empresas.

Para las agencias de viaje, el análisis de las entrevistas muestra que de acuerdo con la tabla 1, las fuentes de conocimiento que tienen como origen la investigación, no son consideradas por los empresarios, en tanto solo una empresa hizo mención a las bases de datos, ver figura 1. Esto es una dificultad si se tiene en cuenta que las universidades y unidades de transferencia, entre otras, tienen como rol esencial la generación y transferencia de conocimiento al sector productivo y no se están aprovechando. Adicionalmente, las fuentes de conocimiento más utilizadas son las propias: empresario o gerente, y las de mercado: proveedores, clientes y competidores; estos hallazgos son consistentes con los planteamientos de Ruizalba, Vallespín y Pérez (2015), sobre la importancia de la información y conocimiento que fundamenta la labor del gerente o propietario, junto con lo indicado por García (2009), sobre la necesidad de obtener información del mercado en tiempo real.

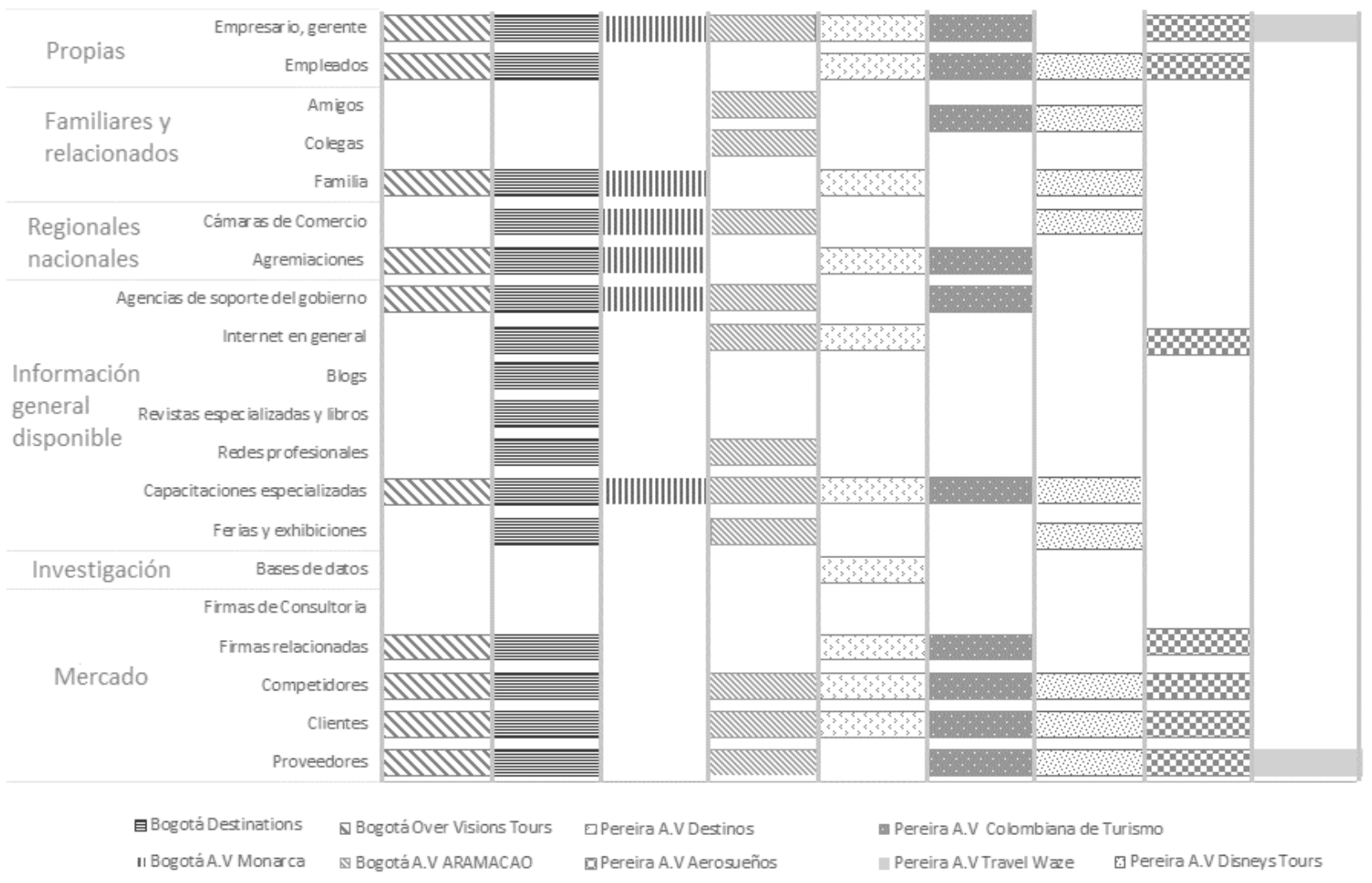

Figura 1. Fuentes de conocimiento en agencias de viaje. 
Por otra parte, se observan diferencias entre Bogotá y Pereira: en la primera, al estar la muestra representada por empresas familiares, el conocimiento que la familia aporta se evidencia en mayor medida que en Pereira. Así mismo, Bogotá a pesar de tener una empresa menos en la muestra, en sumatoria tiene más referencias a fuentes de conocimiento que Pereira, mostrando de esta forma una posible relación entre el tamaño de la ciudad y la diversidad de fuentes a las que se puede acceder. En contraste, al tener Pereira en la muestra empresas más grandes que Bogotá, se torna relevante para estas el conocimiento que aporta el empleado.
En lo que hace referencia a las fuentes de conocimiento de los hoteles, a pesar de pertenecer a la cadena del turismo, su comportamiento difiere en algunos aspectos; por ejemplo, se acentúa el problema de la no utilización de fuentes relacionadas con la investigación, ya que ninguno de los empresarios las mencionó, y tanto las propias -empleados-, como las de mercado -competidores-, son parcialmente utilizadas, ver figura 2 . En este caso, los empleados no son una fuente importante de conocimiento, pero sí la familia del empresario.

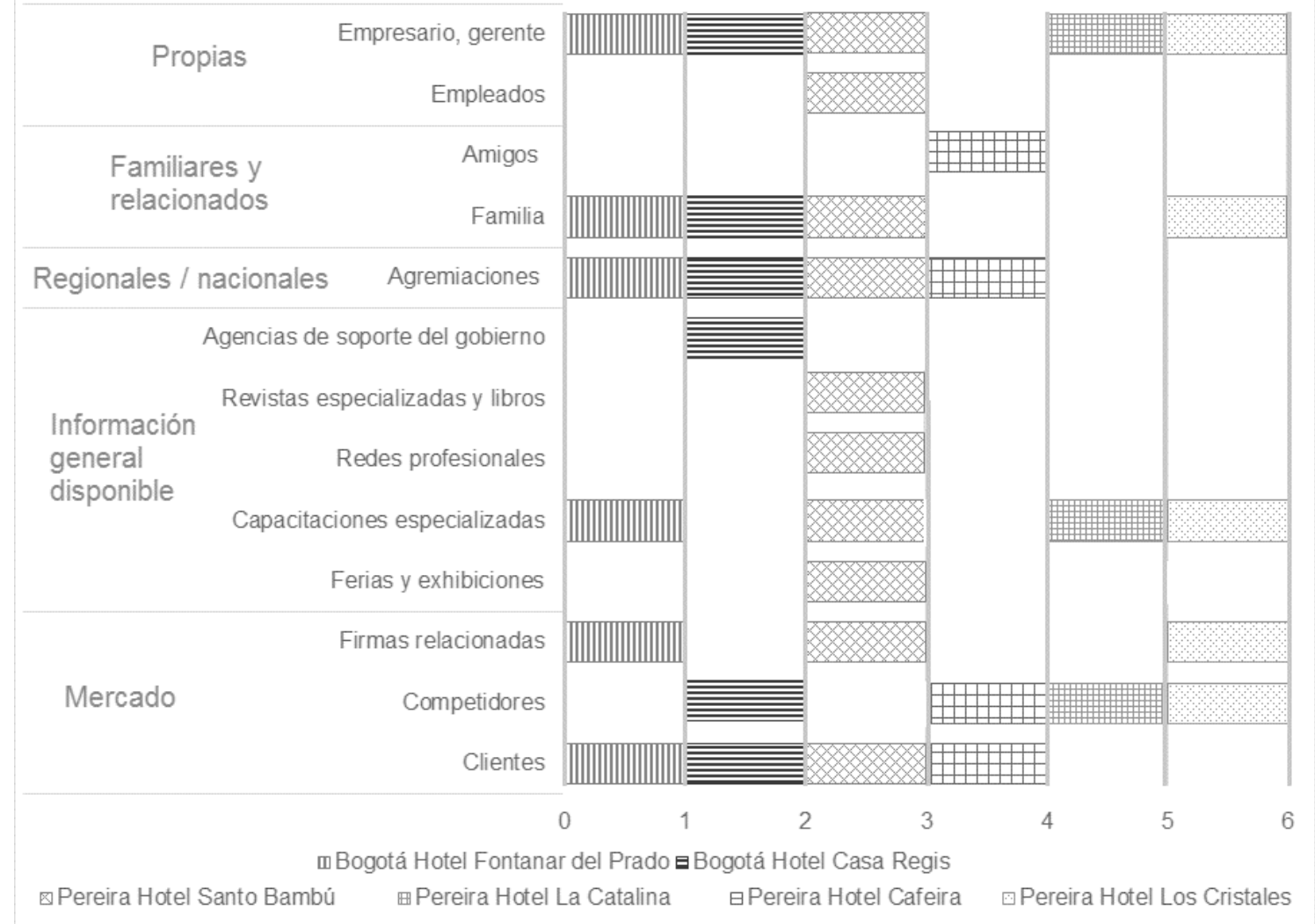

Figura 2. Fuentes de conocimiento hoteles.

En general, en ambas ciudades, el uso de las fuentes de conocimiento a excepción de un hotel, es más escaso que lo observado con las agencias de viaje, posiblemente asociado al tamaño de las em- presas, pero también a que es un subsector menos dinámico. Es evidente que en este caso, la fuente primordial de conocimiento es el gerente. 
3.2 Imágenes sobre la innovación en las empresas estudiadas

El contexto empresarial es el escenario donde se encuentran diversos modos de observar, interpretar e intervenir en la realidad. Así se van configurando las prácticas de las organizaciones, que comprenden, entre muchas otras, las que se relacionan con la generación de innovaciones. La actividad empresarial, como todos los tipos de actividad humana, está permeada por una serie de intereses, imágenes y sistemas de creencias que van determinando las trayectorias de los empresarios.

Se retomaron los planteamientos de Morales y Rizo (1999) para quienes las imágenes son ideas o nociones que están presentes de modo consciente en los individuos y que constituyen una forma de conocimiento específico, una modalidad de pensamiento práctico, orientado a caracterizar los objetos y a relacionarlos con los contextos específicos.

Desde esta perspectiva las imágenes de la innovación que se encontraron en los empresarios hoteleros se refieren a marcar la diferencia, a hacer cosas distintas que sorprendan a los clientes. En algunos casos para proponer una definición se recurre a los ejemplos y estos se relacionan con la mercadotecnia y con la creación de nuevos productos.

Las agencias de viajes tienen una mayor riqueza en las concepciones sobre innovación, lo que permite evidenciar categorías emergentes en cuatro grandes dimensiones: 1) Prácticas diferenciadoras en la prestación del servicio; 2) Novedad, mejoramiento; 3) Apropiación de tecnología; 4) El cliente es el que valida las propuestas de innovación, es decir, es el mercado el que valora y aprueba la innovación.

Las percepciones existentes sobre la innovación en el subsector hotelero se dividen en dos grandes grupos: los que consideran que es un sector muy tradicional, con desarrollos muy estandarizados y con poca diferenciación, y los que identifican una serie de prácticas novedosas referidas a inversión en tecnología para ofrecer servicios que representen un mayor confort para el cliente; identificación de nichos de mercado; explotación de ventajas comparativas referidas, por ejemplo, al sitio donde están ubicados.

En el subsector de las agencias de viajes es mayoritaria la concepción de que hay prácticas de innovación en este tipo de empresas, y están referidas especialmente a tecnología como: la utilización de canales de comunicación con el cliente o al desarrollo de la actividad a través de plataformas tecnológicas. También se considera que se puede hacer innovación de producto, aunque desafortunadamente es una ventaja de muy corta duración por la cultura de algunos empresarios, de imitar y copiar lo que hacen otros.

Al indagar sobre el desarrollo de innovaciones en cada una de las empresas entrevistadas, las respuestas se encuentran asociadas a la noción de introducir cambios, cualquier tipo de cambio, aunque esto no represente novedad para los clientes, ni para el sector en general. Se puede identificar aquí una disonancia entre la definición de innovación en forma estricta y la práctica cotidiana de la misma.

Sin embargo, como se mencionó anteriormente, para el Manual de Oslo la exigencia mínima para reconocer una innovación es el hecho de ser nueva para la empresa; en este sentido, innovación se consideran las adecuaciones en infraestructura y en tecnología, ampliar la oferta de servicios ofrecidos, o capacitar al personal en temas específicos que permitan ofrecer un mejor servicio. Un aspecto a destacar es que en ambos subsectores se menciona la innovación organizacional referida, por un lado, a las características del trabajo en equipo con los colaboradores y por otro, al desarrollo de nuevas formas de trabajo interno que representen una ventaja en el sector. 


\section{Conclusiones}

Los resultados muestran que existen diferencias frente al uso de las fuentes de conocimiento en los dos eslabones de la cadena del turismo. Por un lado, es claro que las agencias de viaje tienen mayor amplitud en sus fuentes de información y conocimiento que los hoteles, lo cual es coherente con la dinámica del subsector; además, para las agencias es en Bogotá, la capital del país, donde los empresarios utilizan más diversidad de fuentes.

Una coincidencia en los dos eslabones de la cadena del turismo estudiadas, es que las fuentes de conocimiento que tienen como origen la investigación, no presentan acogida por parte de los empresarios. Lo anterior hace necesario una mayor sensibilización a las Mipyme para que valoren y comprendan la importancia que tiene buscar conocimiento en las universidades, documentos de propiedad intelectual, bases de datos, entre otras.

Las micro y pequeñas empresas están en interacción con diversas fuentes de información, lo cual les permitiría identificar con mayor precisión los cambios en el entorno y responder frente a ellos. Sin embargo, especialmente para los hoteles se evidenció que la exploración de conocimiento no es deliberada y que aun cuando se reconocen los cambios del entorno, como es el caso del auge de turistas jóvenes que requieren servicios en línea y a través de dispositivos móviles, esto no ha generado todavía cambios en la forma de prestar el servicio. Lo anterior implica que se tienen dificultades en cuanto a la asimilación de conocimiento y aprendizaje organizacional, lo cual es fundamental para el desarrollo de capacidades dinámicas, que permita a las Mipyme ser competitivas en el contexto actual. De esta forma el reto está en buscar un equilibrio entre la amplitud de las fuentes de conocimiento y el aprovechamiento o explotación de las mismas.

La mayoría de las empresas estudiadas son de origen familiar, lo cual hace que sus fuentes fundamentales de conocimiento sean el empresario o dueño, la familia y relacionados. Sin embargo, a medida que se crece en tamaño, los empleados se convierten en fuente de conocimiento y en general se tiene mayor amplitud de fuentes.

Adicionalmente, las imágenes sobre innovación que tienen los empresarios están relacionadas con dimensiones como: prácticas diferenciadoras, novedad o mejora e implementación de herramientas tecnológicas. La innovación, para las empresas estudiadas, consiste en introducir cambios de cualquier tipo, aunque esto no represente novedad para los clientes, ni para el sector en general, basta con que sea nueva para la empresa. En este sentido, la innovación es considerada como las adecuaciones en infraestructura y tecnología; la ampliación de la oferta de servicios ofrecidos o la capacitación al personal en temas específicos que permitan ofrecer un mejor servicio.

Finalmente, se concluye que el uso limitado de las fuentes no logra modificar la base de recursos de manera sustancial, por lo tanto, dificulta la posibilidad de generar innovación en el sentido estricto, pero el uso de prácticas o estrategias diferenciadoras genera unos factores competitivos que permite a las empresas mantenerse en el mercado. Para futuras investigaciones queda abierto el interrogante de: ¿Cuáles serían las estrategias apropiadas para desarrollar capacidades de innovación en Mipyme del sector turismo o sectores con características similares?.

\section{Referencias}

Abeson, F., \& Taku, M. (2009). Knowledge source and small business competitiveness. Competitiveness Review, 12 (2), 88-96.

Acosta, J., \& Fischer, A. (2013). Condiciones de la gestión del conocimiento, capacidad de innovación y resultados empresariales. Un modelo explicativo. Pensamiento y Gestión, 35, 25-63. 
Baba, Y. (2012). Adopting a specific innovation type versus composition of different innovation types: Case study of a Ghanaian bank. International Journal of Bank Marketing, 30 (3), 218-240.

Baregheh, A., Rowley, J., Sambrook, S., \& Davies, D. (2012). Food sector SMEs and innovation types. British Food Journal, 114 (11), 1640-1653.

Cantillo, D. (2011). Un país de Pymes, El Espectador. Recuperado de: http://www.elespectador. com/noticias/economia/un-pais-de-pymes-articulo-285125.

Čiutienè, R., \& Thattakath, E. (2014). Influence of Dynamic Capabilities in Creating Disruptive Innovation. Economics and Business, 26, 15-21.

Confecámaras. (2013). Crece el sector del turismo en Colombia. Recuperado de: http://www.confecamaras.org.co/noticias/237-crece-el-sector-del-turismo-en-colombia

Foro Económico Mundial. (2015). The Travel \& Tourism Competitiveness Report: Growth through Shocks. Ginebra: World Economic Forum.

García, F., Pelechano, E., \& Navas, J. (2007). Las fuentes de conocimiento y la estrategia tecnológica: una cuestión de ajuste. Empresa global y mercados locales. XXI Congreso Anual AEDEM, 67-82. Madrid, España: Universidad Rey Juan Carlos.

García, O. (2009). Retos en la gestión de los viajes de empresa en tiempos difíciles. SAVIA, 64-65.

González, R. (2011). La innovación abierta en las empresas turísticas extremeñas como herramienta de entrada en el turismo de negocios. PASOS, 9 (4), 489-502.

Gros, B., \& Lara, P. (2009). Estrategias de innovación en la educación superior: el caso de la Universitat Oberta de Catalunya. Revista Iberoamericana de
Educación, 49, 223-245. Recuperado de: http:// www.rieoei.org/rie49a09.pdf

Helfat, C., Finkelstein, S., Mitchell, W., Peteraf, M., Singh, H., Teece, D., \& Winter, S. (2007). Dynamic Capabilities: Understanding Strategic Change in Organizations.

Kohler, H., \& González, S. (2014). Elementos para un concepto sociológico de innovación. Empiria. Revista de metodología de ciencias sociales, 29, 6788.

Martínez, A. (2006). Una mirada integral a las capacidades de innovación: el caso de Grupo Court. Recuperado de: http://tesiuami.uam.mx/revistasuam/Denarius/include/getdoc.php?id=404\&article $=156 \&$ mode $=$ pdf

Morales M., \& Rizo N. (1999). Marco teórico referativo de interpretación del desarrollo de la ciencia y la tecnología en Cuba. Memorias del Taller Internacional Ciencia Tecnología Sociedad. Camagüey, Cuba.

OCDE., \& EUROSTAT. (2006). Manual de Oslo: Guía para la recogida e interpretación de datos sobre innovación. París, Francia: Tercera Edición.

Organización Mundial del Turismo- OMT. (1998). Introducción al Turismo. Madrid, España.

Othman, M., Amaraa, N., \& Landrya, R. (2012). SME's degree of openness: the case of manufacturing industries. Journal of Technology Management \& Innovation, 7 (1), 88-210.

Peña, D., \& Serra, A. (2013). La práctica de la responsabilidad social empresarial. Estudio de caso en el sector turístico. Innovar, 23 (49), 101-114.

Porter, M. E. (1988). Ventaja competitiva: Creación y sostenimiento de un desempeño superior. México: CECSA. 
Real Academia Española de la Lengua (2015). Innovación. Recuperado de: http://dle.rae.es/?id=LgxOcfV

Robinson, S., \& Anton, H. (2011). Sources of information and cooperation for innovation in Norway. Journal of International Business Research, 10 (2), 91-102.

Rodríguez, A., \& Jiménez, J. (2010). Innovación Docente en los Grados de la EUEE de la Universidad de Sevilla. Nuevas enseñanzas de grado en la escuela universitaria de estudios empresariales de la Universidad de Sevilla. Sevilla, España: Grupo Editorial Universitario.

Rodríguez, J. (2010). La construcción de la capacidad de innovar en el medio universitario. El caso de la Facultad de Ingeniería de la Universidad Central, Colombia. World Engineering Education Forum. Cartagena de Indias, Colombia.

Ruelas, A. (2004). Innovar en mercados emergentes: el paradigma de la T grande. Harvard Business Review- América Latina. 54-62. Recuperado de: http://flcruz.files.wordpress.com/2008/06/innovar-en-mercados-emergentes.pdf

Ruizalba, J., Vallespín, M., \& Pérez, J. (2015). Gestión del conocimiento y orientación al marketing interno en el desarrollo de ventajas competitivas en el sector hotelero. Investigaciones Europeas de Dirección y Economía de la Empresa, 21, 84-92.
Stake, R.E. (2007). Investigación con estudio de casos. Madrid, España, Ediciones Morata.

Takeuchi, H. (2013). Knowledge-Based View of Strategy. Universia Business Review, (40), 68-79.

Velasco, E., Zamanillo, I., \& Intxaurburu, M. (2007). Evolución de los modelos sobre el proceso de innovación: Desde el modelo lineal hasta los sistemas de innovación. Decisiones basadas en el conocimiento y en el papel social de la empresa: XX Congreso anual de AEDEM, 2, 1-15.

Viega, L. (2011). Innovación y competitividad. Hoy \& Ayer. Revista Antiguos Alumnos del IEEM. 10, 7287. Recuperado de: http://socrates.ieem.edu.uy/ wp-content/uploads/2011/10/hoy_ayer.pdf

Vivas, S. (2013). Implicaciones de las capacidades dinámicas para la competitividad y la innovación en el siglo XXI. Cuad.admon.ser.organ, 26 (47), 119139.

Yew, K., \& Aspinwall, E. (2004). Characterizing knowledge management in the small business environment. Journal of Knowledge Management, 44-62.

Zollo, M., \& Winter, S. (2002). Deliberate Learning and the Evolution of Dynamic Capabilities. Organization Scienc. 